\title{
Progress in the management of neonatal sepsis: the importance of a consensus definition
}

\author{
James L Wynn ${ }^{1}$ and Richard A Polin ${ }^{2}$
}

$\mathbf{N}$ eonatal sepsis is a significant global health problem associated with high mortality and poor long-term outcomes for survivors particularly in under-resourced settings. Unlike adults, who develop sepsis in the late stages of life, survivors of neonatal sepsis experience decades of impairment. No effective treatments exist for sepsis beyond antimicrobials and supportive care. Early recognition and diagnosis are problematic. Positive predictive value and reproducibility of putative diagnostic testing is suboptimal, which leads to a very low clinical suspicion index and high rates of empiric antimicrobial treatment. However, unnecessary exposure to antibacterial agents increases the risk for subsequent short-term pathology including necrotizing enterocolitis and sepsis as well as long-term morbidities including atopy and asthma. Although clinical suspicion is required to detect sepsis, less than $9 \%$ of blood cultures yield a bacterial pathogen (1). These data highlight the ambiguity of clinical signs and limited accuracy of initial clinician suspicion to detect bacterial infection amenable to antibacterial agents. With the benefit of time that reveals the progression of signs in an infected neonate, one can markedly increase the probability of being correct in the suspicion of infection, but a delay in treatment may be deadly. The use of multiple clinical and laboratory definitions to substantiate the presence of sepsis in this population cripples the ability for meaningful investigation and the opportunity to limit the disease and its impact $(1,2)$. The continued use of a variable definition of sepsis means: (i) epidemiologic and long-term impacts of sepsis are difficult if not impossible to accurately assess, (ii) definitive neonatal sepsis is likely to be less common but more devastating than reported, and (iii) accurate diagnostic and prognostic testing will remain out of reach. A positive microbial culture from a normally sterile site (blood) is frequently used as the gold standard to define sepsis. It should be noted that the isolation of a pathogen is not equivalent to the presence of an infection. The converse, however, is also true-the inability to identify a pathogen does not rule out infection. Furthermore, the presence of clinical signs and organ dysfunction that are the result of an acute inflammatory response, especially those that are associated with the isolation of a pathogenic organism from a normally sterile site, cannot be reasonably dissociated from objective evidence of inflammation during the course of the episode.

It is clear that a consensus definition for sepsis that applies to neonates is a critical need. Reducing the impact of this devastating clinical syndrome requires a concerted approach of information exchange among clinicians, epidemiologists, and scientists that is refined by data. Consensus definitions for sepsis align investigators and are used in clinical practice and investigations for all patients with one startling exception: patients cared for in the neonatal intensive care unit (NICU) $(3,4)$. This subset of preterm, high-risk, and physiologically unique newborns requires substantial and prolonged supportive care that is associated with a high risk of infection. In addition to the need for life-saving interventions that increase the risk of infection, distinct and dynamic immunologic function raises the risk for the progression of a localized infection to sepsis.

The use of a Delphi process to get to consensus, test construct validity for specific outcomes, and to validate the definition is dependent upon data. Adult consensus definitions were established in 1991 by expert consensus, revised in 2001 (again by expert consensus), and refined in 2016 using a data-based approach that included six electronic health record databases with nearly 5 million patient encounters (3). Pediatric definitions were established in 2005 (by expert consensus) and have not, as yet, been refined (4). In each of these populations, 12-25 years of consensus definitionderived data (e.g., risk factors, diagnosis, clinical progression, organ failures, mortality, and survivor outcomes) are potentially available to inform and refine the care of patients with sepsis. Despite sepsis being a significant risk associated with prematurity and its treatment as the most commonly prescribed drugs in the NICU, we have not been able to achieve what our adult and pediatric colleagues have accomplished.

The current definition of sepsis in adults is a "lifethreatening organ dysfunction caused by a dysregulated host response to infection" (3). "Life-threatening" is substantiated by the presence of sequential organ dysfunction (objectively

\footnotetext{
${ }^{1}$ Department of Pediatrics, University of Florida, Gainesville, Florida; ${ }^{2}$ Division of Neonatal-Perinatal Medicine, Department of Pediatrics, Columbia University Medical Center, New York City, New York.

Correspondence: James L. Wynn (james.wynn@peds.ufl.edu)

Received 14 August 2017; accepted 29 August 2017; advance online publication 11 October 2017. doi:10.1038/pr.2017.224
} 


\section{Commentary | Wynn and Polin}

determined by the sequential organ failure assessment (SOFA) score) and admission to the intensive care unit with death as a final outcome measure. The presence of organ dysfunction indicates a more complex pathobiology than simply infection with an accompanying inflammatory response. Beyond the obvious differences in age-dependent developmental maturity between adults and children, the patient population in the NICU is distinct from adult and pediatric populations where ICU admission represents a substantial change in the clinical status from baseline. Outcomes such as death and organ dysfunction among patients with suspected infection in the NICU remain valid and should be the basis for a consensus definition for neonatal sepsis based on outcomes. The timing of infection after birth presents another unique conundrum for neonates. Newborns who require critical care support from the time of birth represent a significant challenge to establish a diagnosis of sepsis based on organ dysfunction because there is neither a prior baseline available from which to measure change and developmental changes in organ function, which begin immediately upon delivery. Furthermore, extremely preterm infants commonly require considerable support for reasons unrelated to the infectious process. Neonatal-specific data are limited, but are available to help identify the presence and progression of organ dysfunction that would facilitate the development of a neonatal-specific SOFA (nSOFA, Table 1) like the scoring system used to define sepsis in adults (3). Once a consensus definition is established, data-informed refinement on the accuracy of definition criteria would be expected to improve and incorporate common modifiers of risk in the NICU including gestational age and birth weight.

At present, clinicians caring for critically ill neonates must ultimately determine whether infection and or sepsis is present. The limitation inherent to using a subjective determination that disease is present is that the threshold for declaring sepsis can be markedly different between individual clinicians and investigators, which leads to ambiguity in frequency of disease, outcomes, and diagnostic testing accuracy $(1,2)$. Scientists who study neonatal sepsis should be the first target audience of a consensus definition. Late-onset sepsis should be the target disease to allow identification of progressive organ failure from a baseline. An initial consensus definition must be sufficiently rigorous that it would be unequivocally validated as a case of sepsis on retrospective review. As such, the definition would require the following: (i) a subjective assessment that infection was highly likely (clinician sent cultures and initiated empiric antimicrobial treatment), (ii) objective evidence of organ dysfunction consistent with sepsis, and (iii) objective documentation of systemic inflammation consistent with the biology of an infection during the episode (acute phase response consistent with sepsis). Reporting of clinical signs and laboratory test results must be accompanied by timing relative to original clinical presentation and organ dysfunction. By using a training cohort to identify the presence of sequential organ failure among neonates who died with suspected or confirmed infection (5), one can identify potential criteria to be tested for a consensus definition of sepsis (construct validity, e.g., nSOFA). These clinical criteria would then be subsequently

Table 1. Hypothetical nSOFA

\begin{tabular}{|c|c|c|c|c|}
\hline System & 0 & 1 & 2 & 3 \\
\hline Respiratory & $\begin{array}{l}\text { No support } \\
\text { or } \\
\mathrm{Ol}<2 \\
\mathrm{PaO}_{2} / \mathrm{FiO}_{2}>330 \\
(70 / 0.21)\end{array}$ & $\begin{array}{l}\text { CPAP/HFNC } \\
\text { or } \\
\text { Ol-2-8 } \\
\text { (max of } 40 \% \mathrm{O}_{2} \text {, max MAP 14, } \mathrm{paO}_{2} \\
70) \\
\mathrm{PaO}_{2} / \mathrm{FiO}_{2} 230-330(70 / 0.3)\end{array}$ & $\begin{array}{l}\text { NIPPV } \\
\text { or } \\
\text { Ol-8-14 } \\
\left(\max 60 \% \mathrm{O}_{2} \text {, max MAP }\right. \\
\left.16, \mathrm{paO}_{2} 70\right) \\
\mathrm{PaO}_{2} / \mathrm{FiO}_{2} \quad 140-260 \\
(70 / 0.5)\end{array}$ & $\begin{array}{l}\text { Intubated: } \mathrm{CMV} / \mathrm{HFV} \\
\text { or } \\
\mathrm{Ol}_{-14-20} \\
\mathrm{PaO}_{2} / \mathrm{FiO}_{2}<140(70 / 0.6) \text { or any iNO } \\
\mathrm{Score} \text { of } 4 \text { : ECMO (if eligible), OI }>20 \text { if } \\
\text { ineligible }\end{array}$ \\
\hline Platelets $\left(10^{3} / \mu \mathrm{l}\right)$ & $\geq 100$ & $<100$ & $<50$ & $<50$ in $\leq 24 \mathrm{~h}$ after transfusion \\
\hline 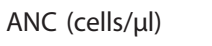 & $>1,500$ & $1,001-1,500$ & $500-1,000$ & $<500$ \\
\hline $\begin{array}{l}\text { Renal } \\
\text { (Cr-mg/dl, UOP } \\
\mathrm{ml} / \mathrm{kg} / \mathrm{h})\end{array}$ & $\begin{array}{l}\text { UOP }>0.5 \\
\text { and } \\
\text { no change in } \mathrm{sCr} \text { or rise } \\
<0.3\end{array}$ & $\begin{array}{l}\text { UOP }<0.5 \text { for } 6-12 \mathrm{~h} \text { and } \\
\mathrm{sCr} \text { increase }>0.3 \text { in } 48 \mathrm{~h} \\
\text { or } \\
>1.5-1.9 \times \text { LPC value within } 7 \text { days }\end{array}$ & $\begin{array}{l}\text { UOP }<0.5 \text { for } \geq 12 \mathrm{~h} \\
\text { and } \\
\geq 2.0-2.9 \times \text { LPC value }\end{array}$ & $\begin{array}{l}\text { UOP }<0.5 \text { for } \geq 12 \mathrm{~h} \\
\text { and } \\
\geq 3 \times \mathrm{LPC} \text { value } \\
\text { or } \\
\mathrm{sCr}>2.5 \\
\text { or } \\
\text { dialysis }\end{array}$ \\
\hline CNS & Baseline responsiveness & Any change in status & Lethargic or hypotonic & Unresponsive \\
\hline
\end{tabular}

CMV, conventional mechanical ventilation; CPAP, continuous positive airway pressure; ECLS, extracorporeal life support; GA, gestational age; HFNC, high-flow nasal cannula; HFV, high-frequency ventilation; LPC, lowest previous SCr; MAP, mean arterial pressure; NIPPV, non-invasive positive pressure ventilation; Ol, oxygenation index; SBP, systolic blood pressure; sCr, serum creatinine; UOP, urine output. 


\section{Commentary | Commentary}

tested in another cohort of patients to determine whether the construct (e.g., nSOFA score) predicts mortality (predictive validity). Predictive clinical criteria (e.g., nSOFA score $>4$ predicts mortality) would then be validated in other cohorts of patients to determine the generalizability of the definition. At each stage (construct validity, predictive validity, and generalizability), a Delphi process using an expert panel would be used and supplemented by extensive literature review. Once a predictive definition is derived, relevant clinical societies would be given the data and the opportunity to endorse the definition with the goal of achieving consensus. Interested professionals with expertise in neonatal sepsis and access to robust neonatal clinical databases, as well as the support and endorsement of societies with members who care for patients in the NICU, are necessary to achieve this goal. We initiated and are committed to the success of this endeavor. However, recognition of the importance of this issue by major funding sources is needed for development and implementation of a consensus definition. The surest way to finish is to start.

Disclosure: The authors declare no conflict of interest.

\section{REFERENCES}

1. Wynn JL. Defining neonatal sepsis. Curr Opin Pediatr 2016;28:135-40.

2. Wynn JL, Wong HR, Shanley TP, Bizzarro MJ, Saiman L, Polin RA. Time for a neonatal-specific consensus definition for sepsis. Pediatr Crit Care Med 2014;15:523-8.

3. Singer M, Deutschman CS, Seymour CW, et al. The third international consensus definitions for sepsis and septic shock (sepsis-3). JAMA 2016;315:801-10.

4. Goldstein B, Giroir B, Randolph A. International pediatric sepsis consensus conference: definitions for sepsis and organ dysfunction in pediatrics. Pediatr Crit Care Med 2005;6:2-8.

5. Wynn JL, Kelly MS, Benjamin DK, et al. Timing of multiorgan dysfunction among hospitalized infants with fatal fulminant sepsis. Am J Perinatol 2017;34:633-9. 